\title{
Pleural thickness after neoadjuvant chemotherapy is a prognostic factor in malignant pleural mesothelioma
}

Masaki Hashimoto, MD, PhD, ${ }^{\mathrm{a}}$ Jiro Takeuchi, MD, PhD, ${ }^{\mathrm{b}}$ Teruhisa Takuwa, MD, PhD, ${ }^{\mathrm{a}}$

Ayumi Kuroda, MD, ${ }^{\mathrm{a}}$ Akifumi Nakamura, MD, ${ }^{\mathrm{a}}$ Toru Nakamichi, MD, ${ }^{\mathrm{a}}$ Seiji Matsumoto, MD, PhD, ${ }^{\mathrm{a}}$ Nobuyuki Kondo, MD, PhD, ${ }^{a}$ Takashi Nakano, $\mathrm{MD}, \mathrm{PhD},{ }^{\mathrm{c}}$ Takeshi Morimoto, $\mathrm{MD}, \mathrm{PhD},{ }^{\mathrm{b}}$ and Seiki Hasegawa, MD, $\mathrm{PhD}^{\mathrm{a}}$

\section{ABSTRACT}

Objectives: Definitive diagnosis of the T-component is sometimes challenging in malignant pleural mesothelioma (MPM). Pleural thickness has been reported to be a prognostic factor for MPM and is a potential T-component.

Methods: We conducted a historical cohort study of patients who underwent neoadjuvant chemotherapy (NAC) and curative-intent surgery as a multimodal treatment for MPM from January 2007 to June 2016. The maximum measurement of pleural thickness among 3 levels and the sum at each level determined using axial computed tomography imaging before and after NAC were termed as "max" and "sum," respectively. We assessed the association between pleural thickness and the primary and secondary end points of overall survival and recurrence-free survival. Survival was analyzed using the Kaplan-Meier curve, log rank test, and multivariate Cox regression model.

Results: We enrolled 105 patients. We excluded 1 because of missing data; thus, the sample size was 104 . The median follow-up period was 29.1 months with recurrence in 78 patients $(70.3 \%)$ and death in $67(60.4 \%)$. Max and sum ranged from pre (before NAC) values of 0 to 35 (median, 6.05) and 0 to 97 (median, 12.9) to post (after NAC) values of 0 to 30.8 (median, 4.25) and 0 to 67.0 (median, 9.25), respectively. Post values max and sum were associated with overall survival and recurrence-free survival. Post sum values were associated with recurrence (adjusted hazard ratio, 2.59; 95\% confidence interval, 1.42-3.83) and death (adjusted hazard ratio, 2.13; 95\% confidence interval, 1.16-4.52), respectively.

Conclusions: Pleural thickness after NAC was an independent prognostic factor in patients who underwent multimodal treatment. (J Thorac Cardiovasc Surg 2019;157:404-13)

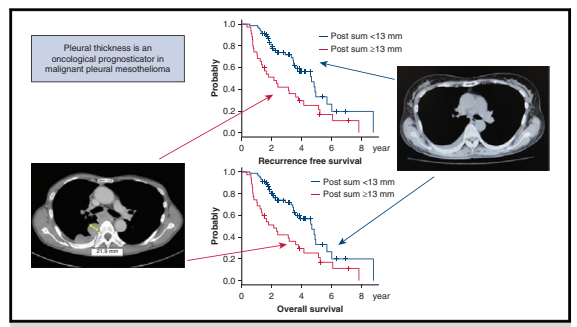

Pleural thickness correlated with oncological outcomes in malignant pleural mesothelioma.

\section{Central Message}

Pleural thickness after neoadjuvant chemotherapy was a novel prognostic factor in a patient who underwent multimodal treatment for malignant pleural mesothelioma.

\section{Perspective}

Pleural thickness has recently been considered as a novel prognostic factor in malignant pleural mesothelioma. In the present study, we evaluated the prognostic meaning of pleural thickness in a patient who underwent multimodal treatment. Pleural thickness was correlated with oncological outcomes better than the current staging systems. Pleural thickness has potential as a new T-component.

See Editorial Commentary page 414.
Tumor diameter is usually associated with aspects determining the tumor status, such as the T-component, in the tumor, node, metastases staging system. However, in

From the Departments of ${ }^{\mathrm{a}}$ Thoracic Surgery and ${ }^{\mathrm{b}}$ Clinical Epidemiology, Hyogo College of Medicine, Nishinomiya, Japan; and ${ }^{c}$ Center for Respiratory Medicine, Otemae Hospital, Osaka, Japan.

This study was presented at the 25th Meeting of European Society of Thoracic Surgeons, May 29, 2017, Innsbruck, Austria.

Received for publication May 11, 2018; revisions received Aug 24, 2018; accepted for publication Sept 11, 2018 .

Address for reprints: Masaki Hashimoto, MD, PhD, Department of Thoracic Surgery, Hyogo College of Medicine, Nishinomiya, Japan (E-mail: kogekogemasaki@ gmail.com).

$0022-5223 / \$ 36.00$

Copyright (c) 2018 by The American Association for Thoracic Surgery

https://doi.org/10.1016/j.jtcvs.2018.09.106 malignant pleural mesothelioma (MPM), the clinical definition of the tumor diameter remains unclear. One of the reasons underlying this concern is that the tumor status has been described according to the extent of tumor infiltration to the surrounding organs. ${ }^{1,2}$ Therefore, the tumor diameter does not influence the current T-component in MPM. In fact, assessing the tumor diameter in MPM is quite

Scanning this QR code will take you to the article title page to access supplementary information.

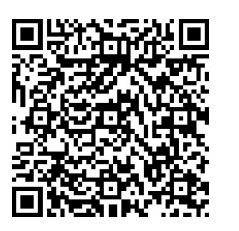




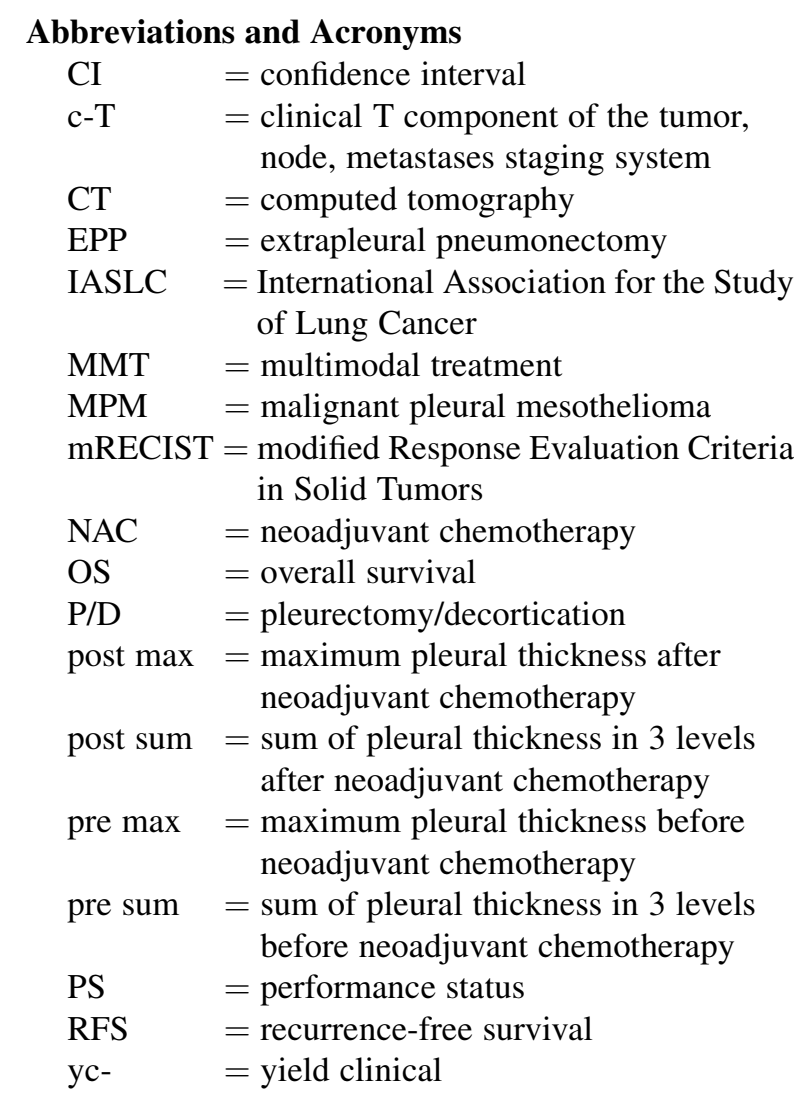

complicated because of its unique development process that differs from that of other solid malignancies.

In the current staging system, there are some unsolved issues, especially those with respect to the T-component. ${ }^{2-5} \mathrm{~A}$ discrepancy between preoperative (clinical) and postoperative (pathological) staging was observed in approximately half of the patients who underwent surgical treatment. ${ }^{5}$ Moreover, the clinical (c)-T component was underevaluated against the pathological (p)-T component in half of the cases despite showing progress in radiological examinations. ${ }^{5}$ In fact, clinical outcomes with a median overall survival (OS) of 12 to 20 months were not satisfactory even after administering multimodal treatment (MMT) because of the aggressive nature of the tumor. ${ }^{6-9}$ Conversely, a favorable prognosis (median OS, 29 months) was observed in p-stage I patients. ${ }^{10}$ Therefore, appropriate patient allocation for MMT plays an important role in the treatment of MPM. ${ }^{11,12}$

Pleural thickness has been recently reported as a useful prognostic indicator of MPM. ${ }^{2,13}$ The International Association for the Study of Lung Cancer (IASLC) revised the definition of MPM staging in 2016 (8th edition) and described that the measurements of pleural thickness were significantly correlated with the clinical outcome. $^{2}$ They also mentioned that pleural thickness might be a useful T-component instead of the pathological findings on the basis of the current T-component in MPM staging. ${ }^{2}$ We hypothesized that pleural thickness was a useful prognostic factor and retrospectively investigated the clinical association between pleural thickness and the prognosis of MPM in our cohort.

\section{METHODS}

\section{Study Design and Patient Selection}

We conducted a historical cohort study to evaluate the clinical effect of pleural thickness in MPM patients who underwent MMT, including curative-intent surgery. We enrolled consecutive patients with histologically proven MPM who underwent MMT, including neoadjuvant chemotherapy (NAC) and curative-intent surgery, at the Hyogo College of Medicine Hospital between January 2007 and June 2016.

\section{Therapeutic Strategy}

As mentioned in the therapeutic guidelines, ${ }^{14}$ curative-intent surgery should be performed as part of MMT in patients with resectable MPM The inclusion criteria for MMT were: (1) histologically proven MPM; (2) radiologically resectable tumor (clinical [c-]stage, T0-3); (3) administration of MMT being feasible with a favorable performance status (PS); (4) being tolerant to curative-intent surgery; and (5) not proven N2-3.

Overall, NAC was performed before curative-intent surgery in patients who met the previously mentioned inclusion criteria. After completion of

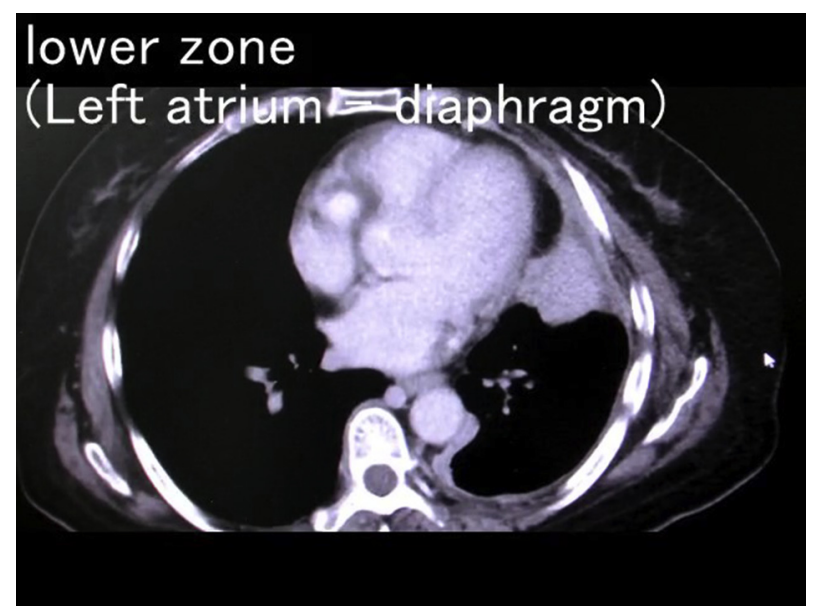

VIDEO 1. We propose pleural thickness as a novel prognostic factor in malignant pleural mesothelioma. It is well known that the current T-component is not satisfactory to predict the clinical outcome in malignant pleural mesothelioma. In this cohort, we clarified that the efficacy of measurement of pleural thickness after neoadjuvant chemotherapy was a better prognosticator than the current T-component. Our measurement procedure is quite simple, and could be used throughout the world. I am going to introduce our measurement procedure. As you can see, we divided the thoracic cavity into 3 zones as upper, middle, and lower. Then, using axial imaging, the maximum pleural thickness was measured perpendicular from the chest wall or mediastinum in each zone. In our cohort the sum of pleural thickness in 3 zones was a novel prognostic factor Because this procedure does not require any specific device or software, it has potential as a new T-component. Video available at: https://www. jtcvs.org/article/S0022-5223(18)32615-1/fulltext. 
NAC, radiological examinations were performed to evaluate the response to NAC on the basis of the modified Response Evaluation Criteria in Solid Tumors (mRECIST). ${ }^{15}$

Curative-intent surgery including lymph node dissection was performed in patients who showed no apparent tumor progression. Extrapleural pneumonectomy (EPP) was the only surgical procedure that we performed before September 2012. After September 2012, we attempted to perform pleurectomy/decortication (P/D) in all patients, and EPP was performed when it was the only procedure that could help us achieve macroscopic complete resection. After completion of curative-intent surgery, adjuvant radiation therapy was only delivered after EPP, and adjuvant chemotherapy was only delivered after P/D as well.

\section{Data Collection and Clinical Follow-up After MMT}

Medical records of all eligible patients, including operation notes and radiological findings, were collected. The c-stage, yield clinical (yc)-stage, and p-stage were determined using the Seventh Edition of the Tumor, Node, Metastases Classification proposed by the International Mesothelioma Interest Group and IASLC. ${ }^{1}$ We excluded cases in which reliable data pertaining to the radiological examination were missing.

Clinical follow-up after MMT was performed at least every 6 months with positron emission tomography/computed tomography (CT) or thin-section CT for as long as possible. Recurrence was determined by a multidisciplinary team according to radiological findings or on the basis of cytological or pathological examination.

\section{Measurement of Pleural Thickness (Video 1)}

The measurement of pleural thickness was determined using thinsection contrast-enhanced CT by 2 thoracic surgeons experienced in treating MPM. The procedure for measuring pleural thickness described in the IASLC Mesothelioma staging project was followed. ${ }^{2}$ In brief, pleural thickness was measured using axial CT images, with the hemithorax divided into 3 levels (upper, middle, and lower); these thirds were defined as follows: the upper level extended from the apex of the lung to the inferior margin of the aortic arch, the middle level included the pleura between the upper and lower levels, and the lower level was the pleura, including an inferior to the first image on which the left atrium is seen. ${ }^{2}$ Next, the maximum tumor thickness perpendicular to the chest wall or mediastinum was measured using axial imaging at each of the 3 levels (Figure 1). We measured the maximum pleural thickness among the 3 levels and the sum of the pleural thickness at each of the 3 levels before and after NAC in each patient. We defined pre max as maximum pleural thickness before neoadjuvant chemotherapy, post max as maximum pleural thickness after neoadjuvant chemotherapy, pre sum as sum of pleural thickness in 3 levels before neoadjuvant chemotherapy, and post sum as sum of pleural thickness in 3 levels after neoadjuvant chemotherapy.

\section{Statistical Analysis}

The primary aim of this study was to evaluate the association between pleural thickness and OS and investigate the most effective way to evaluate pleural thickness as well as its association with recurrence-free survival (RFS). To evaluate the prognostic significance of pleural thickness, we divided the cohort into 2 groups with a cut off point of max as $5.1 \mathrm{~mm}$ $(<5.1 \mathrm{~mm}$ vs $\geq 5.1 \mathrm{~mm})$ and of sum as $13 \mathrm{~mm}(<13.0 \mathrm{~mm}$ vs $\geq 13.0 \mathrm{~mm}$ ) according to a previous definition of pleural thickness. ${ }^{2}$ RFS was defined as the time from the day of surgery to the day of recurrence; patients without recurrence were censored at the time when they were confirmed to show no recurrence. OS was defined as the time from the day of diagnosis to the day of death, and patients who were still alive were censored at the time when they were confirmed to be alive. We also used linear regression models to evaluate the association between max and sum. The Kaplan-Meier method was used to assess OS and RFS, and the log rank test was used to compare the differences between them. The prognostic significance of pleural thickness was assessed using multivariate Cox proportional hazards model with preoperative variables, including pleural thickness and T-component.

All statistical analyses were performed using EZR (Saitama Medical Center, Jichi Medical University, Saitama, Japan), which is a graphical user interface for R (The R Foundation for Statistical Computing, Vienna, Austria). Two-tailed $P$ values $<.05$ were considered statistically significant.

\section{Ethics}

The institutional review board at the Hyogo College of Medicine (number 2857) approved this study on February 7, 2018, and written informed consent was obtained from all patients. We collected the medical records of all enrolled patients, including operation notes and radiological findings.

\section{RESULTS \\ Patient Characteristics}

Overall, 105 consecutive patients with histologically proven MPM who underwent MMT, including NAC and
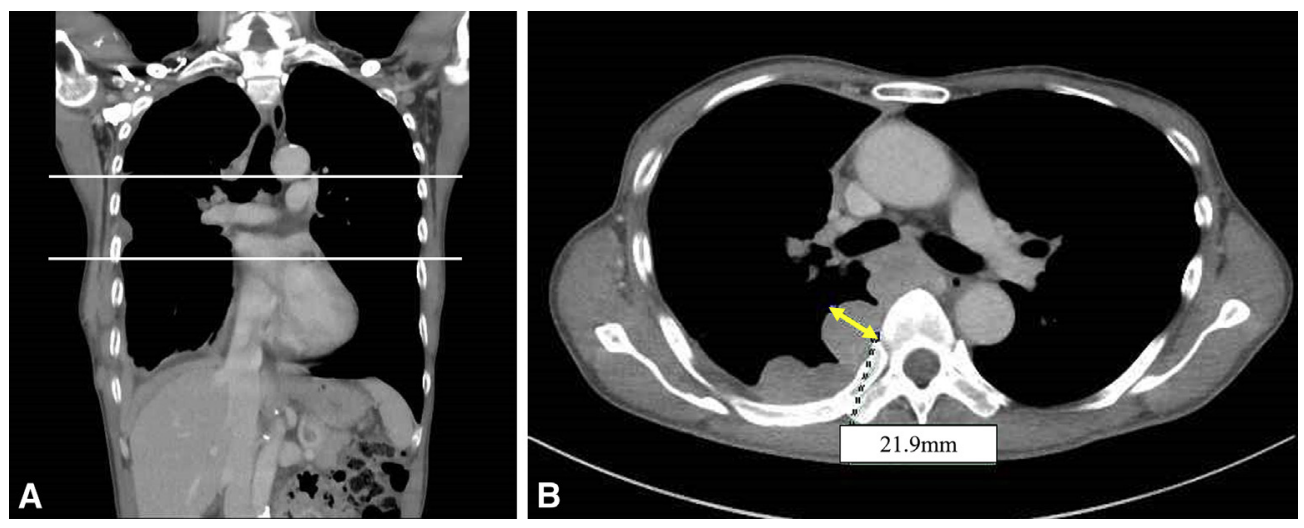

FIGURE 1. The thoracic cavity was divided into 3 levels (upper, middle, and lower). Each level was defined as follows. The upper level was defined as from the apex of the lung to the inferior margin of the aortic arch; the middle level as between the upper and lower levels; and the lower level from an inferior to the first image on which the left atrium is seen (A). Pleural thickness was measured perpendicular to the chest wall or mediastinum for each of the 3 levels on axial imaging (B). Double-headed arrow indicates the measurement of pleural thickness. 
TABLE 1. Patient characteristics $(N=104)$

\begin{tabular}{|c|c|}
\hline Characteristic & Value \\
\hline Median age (range), y & $64(37-77)$ \\
\hline Male sex & $90(86.5 \%)$ \\
\hline \multicolumn{2}{|l|}{ Affected side } \\
\hline Right & $58(55.8 \%)$ \\
\hline Left & $46(44.2 \%)$ \\
\hline \multicolumn{2}{|l|}{ PS } \\
\hline 0 & $97(93.3 \%)$ \\
\hline 1 & $7(6.7 \%)$ \\
\hline \multicolumn{2}{|l|}{ Histological type } \\
\hline Epithelioid & $98(94.2 \%)$ \\
\hline Nonepithelioid & $6(5.8 \%)$ \\
\hline \multicolumn{2}{|l|}{ Clinical stage } \\
\hline I & $53(51.0 \%)$ \\
\hline II & $29(27.9 \%)$ \\
\hline III & $17(16.3 \%)$ \\
\hline IV & $5(4.8 \%)$ \\
\hline \multicolumn{2}{|l|}{ Clinical T component } \\
\hline 1 & $55(52.9 \%)$ \\
\hline 2 & $29(27.9 \%)$ \\
\hline 3 & $18(17.3 \%)$ \\
\hline 4 & $2(1.9 \%)$ \\
\hline \multicolumn{2}{|l|}{ Yield clinical stage } \\
\hline I & $48(46.2 \%)$ \\
\hline II & $37(35.6 \%)$ \\
\hline III & $19(18.3 \%)$ \\
\hline \multicolumn{2}{|c|}{ Yield clinical $\mathrm{T}$ component } \\
\hline 1 & $50(48.1 \%)$ \\
\hline 2 & $38(36.5 \%)$ \\
\hline 3 & $16(15.4 \%)$ \\
\hline \multicolumn{2}{|c|}{ Pathological T component } \\
\hline 1 & $24(23.1 \%)$ \\
\hline 2 & $21(20.2 \%)$ \\
\hline 3 & $52(50 \%)$ \\
\hline 4 & $7(6.7 \%)$ \\
\hline \multicolumn{2}{|l|}{ Nodal involvement } \\
\hline Negative & $74(71.2 \%)$ \\
\hline Positive & $30(28.8 \%)$ \\
\hline \multicolumn{2}{|l|}{ mRECIST } \\
\hline PR & $19(18.3 \%)$ \\
\hline $\mathrm{SD}$ & $84(80.8 \%)$ \\
\hline PD & $1(0.9 \%)$ \\
\hline \multicolumn{2}{|l|}{ Pre max } \\
\hline$<5.1 \mathrm{~mm}$ & $47(45.2 \%)$ \\
\hline$\geq 5.1 \mathrm{~mm}$ & $57(54.8 \%)$ \\
\hline \multicolumn{2}{|l|}{ Post max } \\
\hline$<5.1 \mathrm{~mm}$ & $63(60.6 \%)$ \\
\hline$\geq 5.1 \mathrm{~mm}$ & $41(39.4 \%)$ \\
\hline \multicolumn{2}{|l|}{ Pre sum } \\
\hline$<13 \mathrm{~mm}$ & $52(50.0 \%)$ \\
\hline
\end{tabular}

TABLE 1. Continued

\begin{tabular}{lc}
\hline \multicolumn{1}{c}{ Characteristic } & Value \\
\hline$\geq 13.0 \mathrm{~mm}$ & $52(50.0 \%)$ \\
Post sum & \\
$<13 \mathrm{~mm}$ & $69(66.3 \%)$ \\
$\geq 13.0 \mathrm{~mm}$ & $35(33.7 \%)$ \\
\hline
\end{tabular}

Data are presented as $\mathrm{n}(\%)$ except where otherwise stated. $P S$, Performance status; mRECIST, modified Response Evaluation Criteria in Solid Tumors; PR, partia response; $S D$, stable disease; $P D$, progressive disease; pre max, maximum pleural thickness before neoadjuvant chemotherapy; post max, maximum pleural thickness after neoadjuvant chemotherapy; pre sum, sum of pleural thickness in 3 levels before neoadjuvant chemotherapy; post sum, sum of pleural thickness in 3 levels after neoadjuvant chemotherapy.

curative-intent surgery, were included; 1 patient was excluded from analysis because of missing reliable data pertaining to the radiological findings. No patient was excluded because of missed follow-up. Patient characteristics are shown in Table 1.

The median patient age was 64 (range, 37-77) years, and most were those with PS $0(93.3 \%)$ and male sex $(86.5 \%)$. Histological findings revealed that most patients were of the epithelioid type $(94.2 \%)$. The median cycle of NAC was 3 (range, 1-6). EPP was applied in 49 patients $(47.1 \%$ ), and $\mathrm{P} / \mathrm{D}$ was used in $55(52.9 \%)$. Adjuvant radiation therapy was delivered in 37 of 49 patients who underwent EPP. Adjuvant chemotherapy was delivered in 49 of 55 patients who underwent P/D. With the median follow-up period of 29.1 (range, 4.4-107.0) months, 71 patients $(68.3 \%)$ had tumor recurrence at the last follow-up. Of the $60(57.7 \%)$ patients who died, 9 patients died without the evidence of tumor recurrence.

\section{Measurement of Pleural Thickness and Correlation Between Max and Sum}

Pleural thickness was measured before and after NAC. The pre max and sum values ranged from 0 to $35 \mathrm{~mm}$ (median, $6.05 \mathrm{~mm}$ ) and 0 to $97 \mathrm{~mm}$ (median, $12.9 \mathrm{~mm}$ ), respectively. The post max and sum values ranged from 0 to $30.8 \mathrm{~mm}$ (median, $4.25 \mathrm{~mm}$ ) and 0 to $67 \mathrm{~mm}$ (median, $9.25 \mathrm{~mm}$ ), respectively. Significant correlations were observed between pre max and sum $(r=0.94)$ and post max and sum $(r=0.95)$ values (Figure 2).

\section{Prognostic Analysis Between Pleural Thickness and RFS}

Kaplan-Meier curves for RFS on the basis of pleural thickness are shown in Figures 3 and 4. Regarding pleural thickness before NAC (Figure 3), pre sum values were significantly correlated with RFS (pre sum: $<13 \mathrm{~mm}$, median RFS: 23.62 months [95\% confidence interval (CI), 19.31-34.92] vs pre sum: $\geq 13 \mathrm{~mm}$, median RFS: 13.83 months [95\% CI, 8.25-24.05], $P=.02$ ) and no 


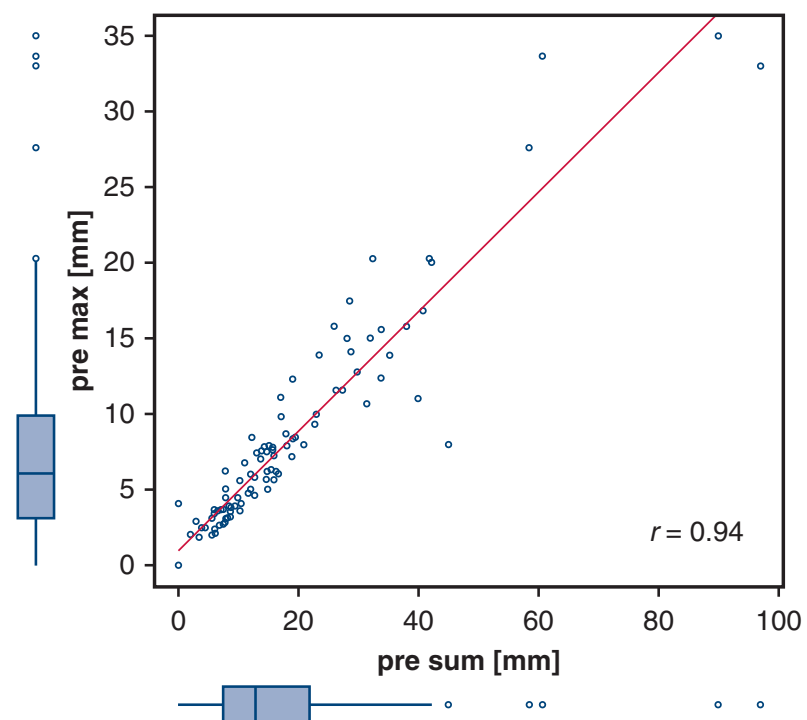

A

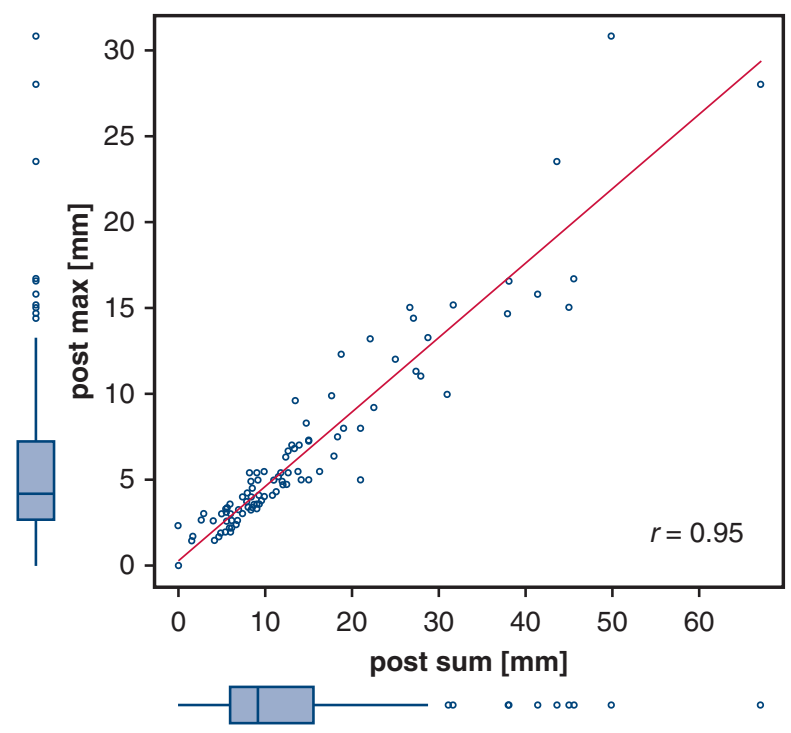

B

FIGURE 2. The correlation between maximum pleural thickness (max) and sum (A) pre and (B) post neoadjuvant chemotherapy. pre max, Maximum pleural thickness before neoadjuvant chemotherapy; pre sum, sum of pleural thickness in 3 levels before neoadjuvant chemotherapy; post max, maximum pleural thickness after neoadjuvant chemotherapy; post sum, sum of pleural thickness in 3 levels after neoadjuvant chemotherapy.

significant correlation was observed between pre max values and RFS. Regarding pleural thickness after NAC (Figure 4), a significant correlation was observed between RFS and post max values (post max: $<5.1 \mathrm{~mm}$, median RFS: 23.62 months [95\% CI, 19.32-36.60] vs pre max: $\geq 5.1 \mathrm{~mm}$, median RFS: 15.38 months [95\% CI, 6.83$22.51], P<.01$ ) and post sum values (post sum: $<13 \mathrm{~mm}$, median RFS: 25.10 months [95\% CI, 19.32-36.60] vs pre

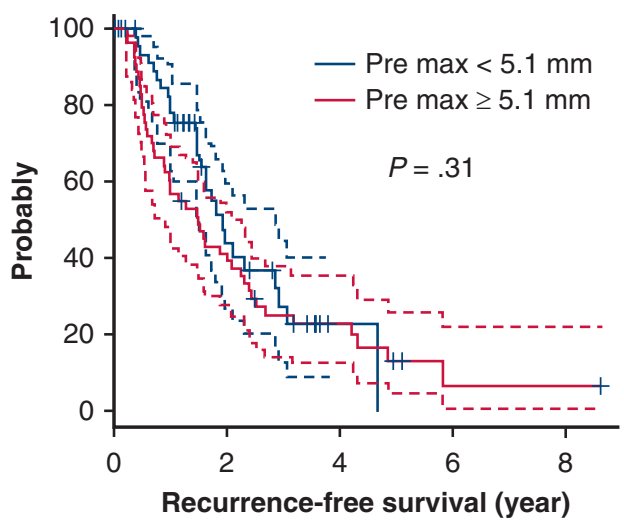

Number at risk

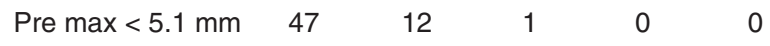

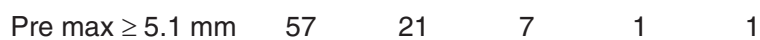

A

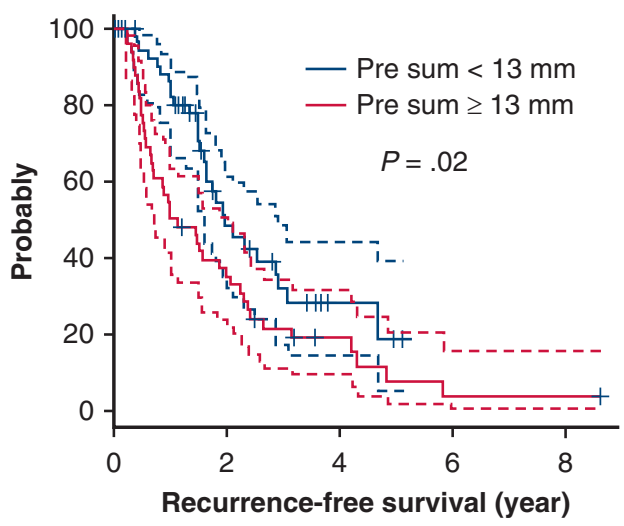

Number at risk

$\begin{array}{llllll}\text { Pre sum }<13 \mathrm{~mm} & 52 & 16 & 3 & 0 & 0 \\ \text { Pre sum } \geq 13 \mathrm{~mm} & 52 & 17 & 5 & 1 & 1\end{array}$

\section{B}

FIGURE 3. Survival curves for recurrence-free survival according to pleural thickness before neoadjuvant chemotherapy. A, Maximum pleural thickness before neoadjuvant chemotherapy (pre max). B, pre sum. pre sum, Sum of pleural thickness in 3 levels before neoadjuvant chemotherapy.

sum: $\geq 13 \mathrm{~mm}$, median RFS: 15.38 months [95\% CI, 8.51-18.99], $P<.001)$.

\section{Prognostic Analysis Between Pleural Thickness and OS}

Kaplan-Meier curves for OS on the basis of pleural thickness are shown in Figures 5 and 6. Regarding pleural thickness before NAC (Figure 5), neither pre max nor pre sum values were correlated with OS. Regarding pleural thickness after NAC (Figure 6), a significant correlation was observed between OS and post max (post max: $<5.1 \mathrm{~mm}$, median OS: 55.20 months [95\% CI, 41.59-68.30] vs post max: $\geq 5.1 \mathrm{~mm}$, median OS: 22.37 months [95\% CI, 14.85-45.67], $P<.01$ ) and post sum (post sum: $<13 \mathrm{~mm}$, 


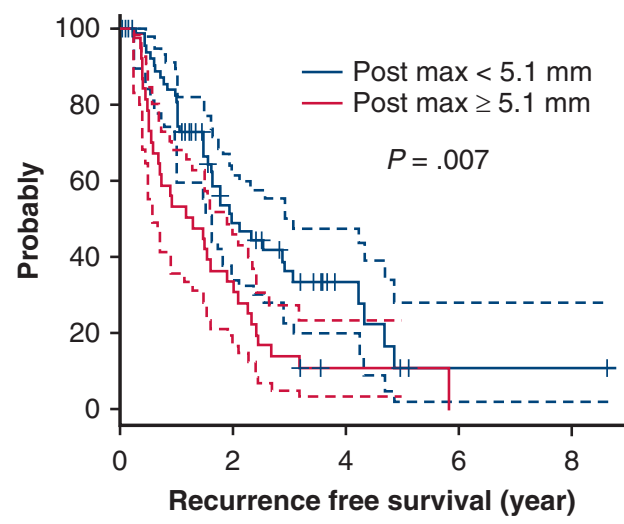

Number at risk

$\begin{array}{llllll}\text { Post } \max <5.1 \mathrm{~mm} & 63 & 21 & 6 & 1 & 1\end{array}$

Post $\max \geq 5.1 \mathrm{~mm} \quad 41 \quad 12 \quad 2 \quad 000$

A

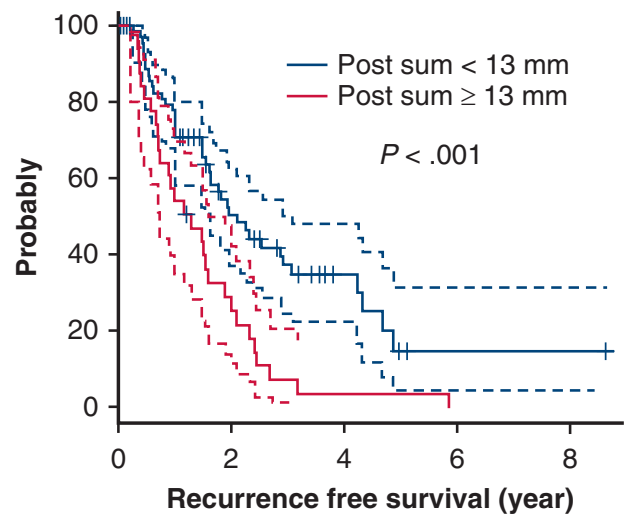

Number at risk

$\begin{array}{llllll}\text { Post sum }<13 \mathrm{~mm} & 69 & 25 & 7 & 1 & 1\end{array}$

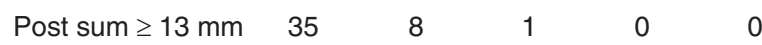

B

FIGURE 4. Survival curves for recurrence-free survival according to (A) maximum pleural thickness after neoadjuvant chemotherapy (post max) and (B) post sum. post sum, Sum of pleural thickness in 3 levels after neoadjuvant chemotherapy.

median OS: 56.21 months [95\% CI, 41.59-68.30] vs post sum: $\geq 13 \mathrm{~mm}$, median OS: 25.86 months $[95 \% \mathrm{CI}$, 14.88-43.33], $P<.01$ ) values.

\section{Prognostic Significance of Pleural Thickness}

The prognostic significance of the preoperative variables is shown in Table 2. Regarding RFS, a significant correlation was observed in the histological types (epithelioid vs nonepithelioid, $P<.0001$ ) and nodal involvement (negative vs positive, $P=.01)$. No significant correlation was observed in c-T component (c-T1 vs c-T2-4, $P=.32$ ), ycT component (yc-T1 vs yc-T2-4, $P=.11$ ), and p-T component (p-T1 vs p-T2-4, $P=.22$ ). Regarding OS, a significant correlation was observed among the histological types (epithelioid vs nonepithelioid, $P<.0001$ ), nodal involvement (negative vs positive, $P<.001)$ and PS $(0$ vs 1 ,

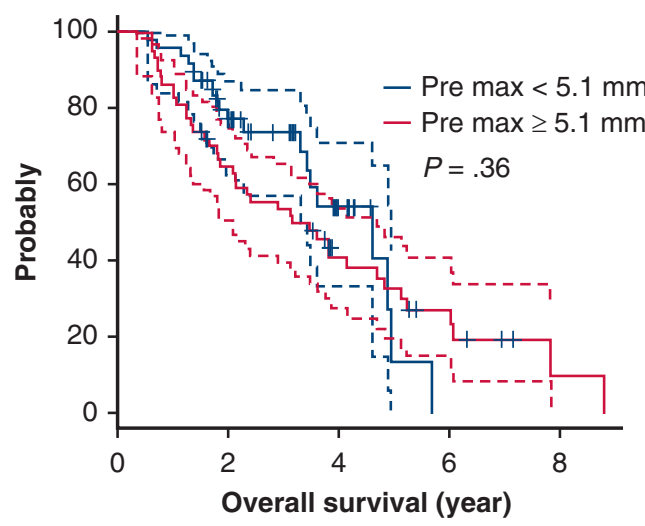

Number at risk

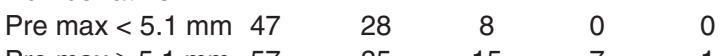

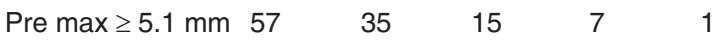

A

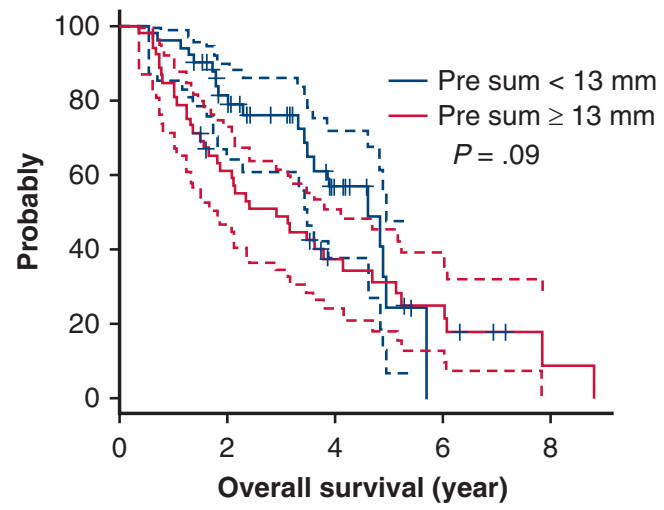

Number at risk

$\begin{array}{llllll}\text { Pre sum }<13 \mathrm{~mm} & 52 & 33 & 11 & 0 & 0\end{array}$

$\begin{array}{llllll}\text { Pre sum } \geq 13 \mathrm{~mm} & 52 & 30 & 12 & 7 & 1\end{array}$

\section{B}

FIGURE 5. Survival curves for overall survival according to (A) maximum pleural thickness before neoadjuvant chemotherapy (pre max) and (B) pre sum. pre sum, Sum of pleural thickness in 3 levels before neoadjuvant chemotherapy.

$P=.01)$. Among T-component, only the $\mathrm{p}-\mathrm{T}$ component was significantly correlated with OS (p-T1 vs p-T2-4, $P<.001)$.

The multivariate Cox model for the post sum value of $\geq 13 \mathrm{~mm}$ showed a significantly increased risk of recurrence (HR, 2.59; 95\% CI, 1.42-4.35; $P<.01)$ and death (HR, 2.13; 95\% CI, 1.16-3.89; $P=.01$ ) (Table 3). Histologic type (nonepithelioid) was also associated with a significantly increased risk of recurrence (HR, $12.81 ; 95 \% \mathrm{CI}$, $4.15-39.57 ; P<.001)$ and death (HR, 5.10; 95\% CI, 1.66$15.63 ; P<.01)$. Positive nodal involvement was also associated with a significantly increased risk of recurrence (HR, $2.22 ; 95 \% \mathrm{CI}, 1.28-3.83 ; P<.01)$ and death $(\mathrm{HR}, 2.54$; $95 \%$ CI, $1.43-4.52 ; P<.01)$. Age $(\geq 65$ years) was associated with a significantly increased the risk of death (HR, $2.12 ; 95 \% \mathrm{CI}, 1.17-3.86 ; P=.01)$ but not of recurrence. 


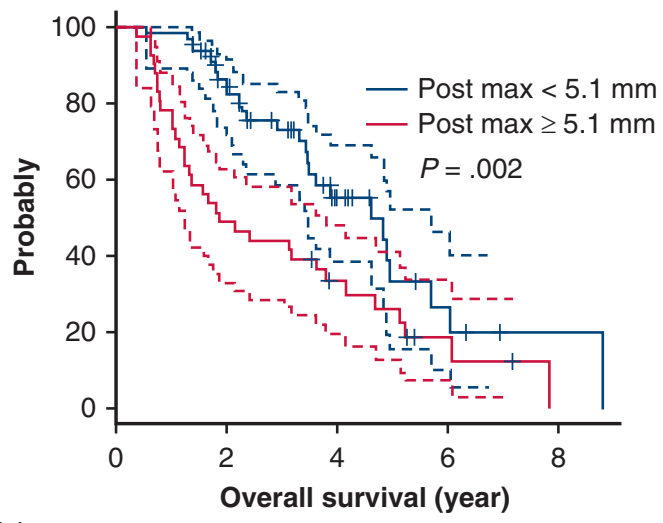

Number at risk

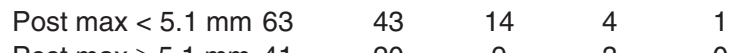

Post $\max \geq 5.1 \mathrm{~mm} 41$

of the 9 sites and showed that the total pleural thickness, which is the sum of all 9 sites, was an independent prognostic factor for RFS as well as OS. In many solid malignancies, we know that a tumor with a small diameter is a more favorable predictor of postoperative prognosis than a tumor with a large diameter. Similar to the results of the study by de Perrot and colleagues, ${ }^{13}$ we showed that pleural thickness was a useful predictor of postoperative outcomes.

In this cohort, pleural thickness was evaluated as max and sum values, which were strongly correlated with before and after NAC. Although max is the easiest measurement procedure to adopt, we speculated that sum would be a better surrogate for tumor volume than max because of the nature of the tumor. MPM generally presents diffuse pleural thickening ${ }^{3,16}$; however, pleural thickness is not uniform. In fact, mRECIST for MPM is evaluated on the basis of the measurement of pleural thickness at 3 points. ${ }^{15}$ Sum might be a favorable measurement procedure for evaluating pleural thickness and will be accepted well globally.

As an alternative procedure for evaluating pleural thickness as a T-component, several studies have reported the utility of tumor volume as a prognostic factor in MPM. ${ }^{16-20}$ Pass and colleagues showed that preoperative tumor volume was a predictor of OS and progression-free survival. ${ }^{20}$ Moreover, Opitz and colleagues supported the efficacy of tumor volume as a prognostic factor and proposed that the multimodality prognostic score was helpful for allocating patients for MMT, including surgery. ${ }^{12}$ The multimodality prognostic score comprised pretreatment tumor volume, C-reactive protein level, and histological subtype in addition to the therapeutic response of NAC according to mRECIST. ${ }^{12}$ Furthermore, several authors have reported that tumor volume was a useful indicator of not only prognosis but also the therapeutic response of chemotherapy. ${ }^{16,19}$ Because 2 multicenter volumetric $\mathrm{CT}$ studies showed volumetric CT was significantly correlated with tumor status $\mathrm{p}-\mathrm{T}$ component and $\mathrm{OS},{ }^{17,18}$ tumor volume is well known as an indicator of tumor status in MPM. However, a specific software is required to measure the tumor volume. In fact, various procedures were performed in the previous cohorts. ${ }^{13,19,20}$ In contrast, our procedure does not require any specific software or any additional costs, suggesting that the measurement of pleural thickness is applicable when describing the T-component worldwide.

In our cohort, pleural thickness after NAC was better correlated with RFS and OS, which suggested that NAC strongly influenced the clinical outcome in patients who underwent MMT. Opitz and colleagues showed that the therapeutic response of NAC was a novel prognostic indicator in MPM patients who were administered MMT. ${ }^{12}$ Our clinical results also revealed a favorable prognosis (median RFS: 25.10 months and median OS: 56.21 months) in patients with post sum values of $<13 \mathrm{~mm}$. These findings suggested that NAC is helpful in identifying the definitive candidates 
TABLE 2. Univariate analyses for RFS and OS

\begin{tabular}{|c|c|c|c|c|c|}
\hline Variable & Patient $n(N=104)$ & Median RFS, months & $P$ value & Median OS, months & $P$ value \\
\hline \multicolumn{6}{|l|}{ Pre max } \\
\hline$<5.1 \mathrm{~mm}$ & 47 & 22.97 & .31 & 55.20 & .36 \\
\hline$\geq 5.1 \mathrm{~mm}$ & 57 & 17.97 & & 38.05 & \\
\hline \multicolumn{6}{|l|}{ Post max } \\
\hline$<5.1 \mathrm{~mm}$ & 63 & 23.62 & $<.01$ & 55.20 & $<.01$ \\
\hline$\geq 5.1 \mathrm{~mm}$ & 41 & 15.38 & & 22.37 & \\
\hline \multicolumn{6}{|l|}{ Pre sum } \\
\hline$<13 \mathrm{~mm}$ & 52 & 23.62 & .02 & 55.20 & .09 \\
\hline$\geq 13 \mathrm{~mm}$ & 52 & 13.83 & & 34.90 & \\
\hline \multicolumn{6}{|l|}{ Post sum } \\
\hline$<13 \mathrm{~mm}$ & 69 & 23.10 & $<.001$ & 56.21 & $<.01$ \\
\hline$\geq 13 \mathrm{~mm}$ & 35 & 15.38 & & 25.86 & \\
\hline \multicolumn{6}{|l|}{ Age } \\
\hline$<65 \mathrm{y}$ & 54 & 19.65 & .52 & 45.57 & .19 \\
\hline$\geq 65 \mathrm{y}$ & 50 & 21.68 & & 41.82 & \\
\hline \multicolumn{6}{|l|}{ Sex } \\
\hline Male & 90 & 20.70 & .84 & 43.33 & .65 \\
\hline Female & 14 & 19.32 & & 58.02 & \\
\hline \multicolumn{6}{|l|}{ Laterality } \\
\hline Left & 46 & 17.64 & .51 & 41.59 & .55 \\
\hline Right & 58 & 21.68 & & 45.57 & \\
\hline \multicolumn{6}{|l|}{ PS } \\
\hline 0 & 97 & 20.70 & .90 & 46.42 & .01 \\
\hline 1 & 7 & 9.48 & & 15.97 & \\
\hline \multicolumn{6}{|l|}{ Histological type } \\
\hline Epithelioid & 98 & 21.68 & $<.0001$ & 46.42 & $<.0001$ \\
\hline Nonepithelioid & 6 & 4.96 & & 14.18 & \\
\hline \multicolumn{6}{|c|}{ Clinical T component } \\
\hline 1 & 55 & 25.10 & .32 & 55.20 & .19 \\
\hline 2 to 4 & 49 & 18.99 & & 38.05 & \\
\hline \multicolumn{6}{|c|}{ Yield clinical $\mathrm{T}$ component } \\
\hline 1 & 50 & 27.82 & .11 & 55.20 & .07 \\
\hline 2 to 4 & 54 & 18.37 & & 37.42 & \\
\hline \multicolumn{6}{|c|}{ Pathological T component } \\
\hline 1 & 24 & 21.68 & .22 & 59.30 & .03 \\
\hline 2 to 4 & 80 & 19.31 & & 41.59 & \\
\hline \multicolumn{6}{|l|}{ mRECIST } \\
\hline PR & 19 & 30.39 & .57 & 49.77 & .23 \\
\hline $\mathrm{SD}+\mathrm{PD}$ & 85 & 19.58 & & 41.82 & \\
\hline \multicolumn{6}{|l|}{ Nodal involvement } \\
\hline Negative & 74 & 23.62 & .01 & 55.20 & $<.001$ \\
\hline Positive & 30 & 12.12 & & 25.00 & \\
\hline
\end{tabular}

RFS, Recurrence-free survival; OS, overall survival; pre max, maximum pleural thickness before neoadjuvant chemotherapy; post max, maximum pleural thickness after neoadjuvant chemotherapy; pre sum, sum of pleural thickness in 3 levels before neoadjuvant chemotherapy; post sum, sum of pleural thickness in 3 levels after neoadjuvant chemotherapy; $P S$, performance status; $m R E C I S T$, modified Response Evaluation Criteria in Solid Tumors; $P R$, partial response; $S D$, stable disease; $P D$, progressive disease.

for MMT, including curative-intent surgery. Although surgery, as a part of MMT, might offer a therapeutic benefit, ${ }^{21}$ we must consider the risk of surgery because of its invasiveness. ${ }^{22-24}$ Therefore, the most essential issue is the appropriate allocation of MPM patients for MMT. Unfortunately, the current T-component is not satisfactory in predicting preoperative prognosis. ${ }^{5,25} \mathrm{We}$ found that pleural thickness was better correlated with prognosis than the conventional T-component (c-T, yc-T, and p-T), suggesting that the former is a novel useful indicator of patient allocation for MMT.

There are limitations inherent to this study design. First, this study was a single-institution historical study with selected patients who underwent MMT. Because our 
TABLE 3. Multivariate analysis for RFS and OS

\begin{tabular}{|c|c|c|c|c|}
\hline \multirow[b]{2}{*}{ Variable } & \multicolumn{2}{|c|}{ RFS } & \multicolumn{2}{|c|}{ OS } \\
\hline & HR $(95 \%$ CI $)$ & $P$ value & HR $(95 \%$ CI $)$ & $P$ value \\
\hline Age $>65$ y & $1.12(0.67-1.85)$ & .67 & $2.12(1.17-3.86)$ & .01 \\
\hline $\begin{array}{l}\text { Histological type } \\
\text { (nonepithelioid) }\end{array}$ & $12.81(4.15-39.57)$ & $<.001$ & $5.10(1.66-15.63)$ & $<.01$ \\
\hline Nodal involvement (positive) & $2.22(1.28-3.83)$ & $<.01$ & $2.54(1.43-4.52)$ & $<.01$ \\
\hline Post sum (>13 mm) & $2.59(1.42-4.35)$ & $<.01$ & $2.13(1.16-3.89)$ & .01 \\
\hline $\mathrm{PS}=1$ & $0.49(0.13-1.87)$ & .30 & $1.87(0.72-4.85)$ & .20 \\
\hline pT-component ( $\mathrm{pT} \geq 2$ ) & $0.69(0.34-1.42)$ & .32 & $1.53(0.59-3.95)$ & .39 \\
\hline
\end{tabular}

$R F S$, Recurrence-free survival; $O S$, overall survival; $H R$, hazard ratio; $C I$, confidence interval; post sum, sum of pleural thickness in 3 levels after neoadjuvant chemotherapy; $P S$, performance status; $p T$, pathologic $\mathrm{T}$ stage.

survival analyses according to pleural thickness was exploratory in nature, a further hypothesis verification would be needed in a prospective study. Second, patients with apparent tumor progression after NAC were excluded from the therapeutic stream of MMT in this cohort, implying that NAC would affect the clinical outcomes as a selection bias. Third, the comparison between pleural thickness and tumor volume was impossible because of the absence of the specific software at our institution. The efficacy of tumor volume was proven to assess the prognosis because it was possible to evaluate the tumor status using 3-dimensional measurements. To clarify the reliability of the measurement of pleural thickness, we plan to conduct a comparative study to assess the association between measurement of pleural thickness and tumor volumetry. Moreover, we have started a prospective nationwide registry to externally validate the implications of pleural thickness. ${ }^{26}$ In this prospective study, pleural thickness (max and sum) was measured in each patient regardless of the therapeutic strategy. The prognostic implications of pleural thickness are clarified through this prospective study.

\section{CONCLUSIONS}

We investigated the meaning of pleural thickness in MPM patients who underwent MMT. Our investigation revealed that pleural thickness after NAC (post sum) was an independent prognostic factor and that lower post sum values $(<13 \mathrm{~mm})$ reduced the risk of recurrence and death in MPM patients who underwent MMT (Central Image). Thus, pleural thickness could be useful for the allocation of MPM patients for MMT. Further prospective nationwide studies are warranted to evaluate pleural thickness as a new T-component in assessment of the prognosis of MPM.

\section{Conflict of Interest Statement}

Seiki Hasegawa has received research funds from Elli Lilly Company, Taiho Pharmaceuticals, and Ono Pharmaceuticals. All other authors have nothing to disclose with regard to commercial support.
The authors thank Miss Risa Murata for secretarial work, and Enago (www.enago.jp) for the English language review.

\section{References}

1. Rusch VW. A proposed new international TNM staging system for malignant pleural mesothelioma. From the International Mesothelioma Interest Group. Chest. 1995; 108:1122-8.

2. Nowak AK, Chansky K, Rice DC, Pass HI, Kindler HL, Shemanski L, et al. The IASLC Mesothelioma Staging Project: proposals for revisions of the T descriptors in the forthcoming eighth edition of the TNM Classification for Pleural Mesothelioma. J Thorac Oncol. 2016;11:2089-99.

3. Nowak AK, Armato SG III, Ceresoli GL, Yildirim H, Francis RJ. Imaging in pleural mesothelioma: a review of imaging research presented at the 9th International Meeting of the International Mesothelioma Interest Group. Lung Cancer. 2010;70:1-6.

4. Gill RR, Gerbaudo VH, Sugarbaker DJ, Hatabu H. Current trends in radiologic management of malignant pleural mesothelioma. Semin Thorac Cardiovasc Surg. 2009;21:111-20.

5. Nakas A, Black E, Entwisle J, Muller S, Waller DA. Surgical assessment of malignant pleural mesothelioma: have we reached a critical stage? Eur J Cardiothorac Surg. 2010;37:1457-63.

6. Hasegawa S, Tanaka F. Malignant mesothelioma: current status and perspective in Japan and the world. Gen Thorac Cardiovasc Surg. 2008; 56:317-23.

7. Opitz I. Management of malignant pleural mesothelioma-The European experience. J Thorac Dis. 2014;6(Suppl 2):S238-52.

8. Rusch VW, Giroux D, Kennedy C, Ruffini E, Cangir AK, Rice D, et al. Initial analysis of the International Association for the Study of Lung Cancer Mesothelioma database. J Thorac Oncol. 2012;7:1631-9.

9. Takuwa T, Hasegawa S. Current surgical strategies for malignant pleural mesothelioma. Surg Today. 2016;46:887-94.

10. Rusch VW, Venkatraman ES. Important prognostic factors in patients with malignant pleural mesothelioma, managed surgically. Ann Thorac Surg. 1999;68: 1799-804.

11. Cao C, Yan TD, Bannon PG, McCaughan BC. Summary of prognostic factors and patient selection for extrapleural pneumonectomy in the treatment of malignant pleural mesothelioma. Ann Surg Oncol. 2011; 18:2973-9.

12. Opitz I, Friess M, Kestenholz P, Schneiter D, Frauenfelder T, Nguyen-Kim TD, et al. A new prognostic score supporting treatment allocation for multimodality therapy for malignant pleural mesothelioma: a review of 12 years' experience. $J$ Thorac Oncol. 2015;10:1634-41.

13. de Perrot M, Dong Z, Bradbury P, Patsios D, Keshavjee S, Leighl NB, et al. Impact of tumour thickness on survival after radical radiation and surgery in malignant pleural mesothelioma. Eur Respir J. 2017;49:1601428.

14. Rusch V, Baldini EH, Bueno R, De Perrot M, Flores R, Hasegawa S, et al. The role of surgical cytoreduction in the treatment of malignant pleural mesothelioma: meeting summary of the International Mesothelioma Interest Group Congress, September 11-14, 2012, Boston, Mass. J Thorac Cardiovasc Surg. 2013;145:909-10.

15. Byrne MJ, Nowak AK. Modified RECIST criteria for assessment of response in malignant pleural mesothelioma. Ann Oncol. 2004;15:257-60. 
16. Ak G, Metintas M, Metintas S, Yildirim H, Ozkan R, Ozden H. Three-dimensional evaluation of chemotherapy response in malignant pleural mesothelioma. Eur J Radiol. 2010;74:130-5.

17. Gill RR, Naidich DP, Mitchell A, Ginsberg M, Erasmus J, Armato SG III, et al. North American Multicenter Volumetric CT Study for Clinical Staging of Malignant Pleural Mesothelioma: feasibility and logistics of setting up a quantitative imaging study. J Thorac Oncol. 2016;11:1335-44.

18. Rusch VW, Gill R, Mitchell A, Naidich D, Rice DC, Pass HI, et al. A multicenter study of volumetric computed tomography for staging malignant pleural mesothelioma. Ann Thorac Surg. 2016;102:1059-66.

19. Frauenfelder T, Tutic M, Weder W, Gotti RP, Stahel RA, Seifert B, et al. Volumetry: an alternative to assess therapy response for malignant pleural mesothelioma? Eur Respir J. 2011;38:162-8.

20. Pass HI, Temeck BK, Kranda K, Steinberg SM, Feuerstein IR. Preoperative tumor volume is associated with outcome in malignant pleural mesothelioma. $J$ Thorac Cardiovasc Surg. 1998;115:310-7; discussion: 7-8.

21. Nelson DB, Rice DC, Niu J, Atay S, Vaporciyan AA, Antonoff M, et al. Long-term survival outcomes of cancer-directed surgery for malignant pleural mesothelioma: propensity score matching analysis. J Clin Oncol. 2017;35:3354-62.
22. Cao C, Tian D, Park J, Allan J, Pataky KA, Yan TD. A systematic review and meta-analysis of surgical treatments for malignant pleural mesothelioma. Lung Cancer. 2014;83:240-5.

23. Sugarbaker DJ, Jaklitsch MT, Bueno R, Richards W, Lukanich J, Mentzer SJ, et al Prevention, early detection, and management of complications after 328 consecutive extrapleural pneumonectomies. J Thorac Cardiovasc Surg. 2004;128:138-46.

24. Flores RM, Pass HI, Seshan VE, Dycoco J, Zakowski M, Carbone M, et al. Extrapleural pneumonectomy versus pleurectomy/decortication in the surgical management of malignant pleural mesothelioma: results in 663 patients. $J$ Thorac Cardiovasc Surg. 2008;135:620-6. 6.e1-3.

25. Rusch VW. Extrapleural pneumonectomy and extended pleurectomy/decortication for malignant pleural mesothelioma: the Memorial Sloan-Kettering Cancer Center approach. Ann Cardiothorac Surg. 2012;1:523-31.

26. Shintani Y, Hasegawa S, Takuwa T, Aoe K, Kato K, Fujimoto N, et al. Prospective registry database of patients with malignant mesothelioma: directions for a future Japanese registry-based lung cancer study. J Thorac Dis. 2018;10:1968-71.

Key Words: malignant pleural mesothelioma, pleural thickness, neoadjuvant chemotherapy 